\title{
Case studies of lightning related injuries and property damage in Zambia
}

\begin{abstract}
This is the first investigation and analysis of lightning related incidents in Zambia. Two case studies on both human injuries and equipment damage at five sites are reported. Lightning injuries were mostly attributed to the lack of proper structural protection systems, although lack of awareness among the public may also contribute to the situation. Many injury mechanisms, including unsuccessful upward streamers, may cause injuries. Design of lightning protection schemes should take social structures and affordability by the affected public into account. Losses in the power sector are excessively high in the region and seriously affect the operation of both business and domestic life. Replacement cost incurred by the power companies can be prohibitive. Lightning density and pattern in the localized areas, grounding systems of the installations, and specification and installation techniques of lightning arresters should be revisited in giving proper solutions to transformer and power line failures.
\end{abstract}

Keyword: Lightning injury; Zambia; Step potential; Direct strikes; Transformer failure; Insulation breakdown 